\title{
A secondary working memory challenge preserves primary place strategies despite overtraining
}

\author{
Robert S. Gardner, ${ }^{1,2,3}$ Michael R. Uttaro, 1,3 Samantha E. Fleming, ${ }^{1,3}$ \\ Daniel F. Suarez, ${ }^{1,2,3}$ Giorgio A. Ascoli, ${ }^{1,2,3}$ and Theodore C. Dumas ${ }^{1,2,3,4}$ \\ ${ }^{1}$ Molecular Neuroscience Department, ${ }^{2}$ Psychology Department, George Mason University, Fairfax, Virginia 22030, USA; \\ ${ }^{3}$ Center for Neural Informatics, Structures, and Plasticity, Krasnow Institute for Advanced Study, George Mason University, \\ Fairfax, Virginia 22030, USA
}

\begin{abstract}
Learning by repetition engages distinct cognitive strategies whose contributions are adjusted with experience. Early in learning, performance relies upon flexible, attentive strategies. With extended practice, inflexible, automatic strategies emerge. This transition is thought fundamental to habit formation and applies to human and animal cognition. In the context of spatial navigation, place strategies are flexible, typically employed early in training, and rely on the spatial arrangement of landmarks to locate a goal. Response strategies are inflexible, become dominant after overtraining, and utilize fixed motor sequences. Although these strategies can operate independently, they have also been shown to interact. However, since previous work has focused on single-choice learning, if and how these strategies interact across sequential choices remains unclear. To test strategy interactions across sequential choices, we utilized various two-choice spatial navigation tasks administered on the Opposing Ts maze, an apparatus for rodents that permits experimental control over strategy recruitment. We found that when a second choice required spatial working memory, the transition to response navigation on the first choice was blocked. Control experiments specified this effect to the cognitive aspects of the secondary task. In addition, response navigation, once established on a single choice, was not reversed by subsequent introduction of a secondary choice reliant on spatial working memory. These results demonstrate that performance strategies interact across choices, highlighting the sensitivity of strategy use to the cognitive demands of subsequent actions, an influence from which overtrained rigid actions may be protected.
\end{abstract}

[Supplemental material is available for this article.]

Whenever a subject engages in a repetitive task or behavior, with practice several aspects of performance undergo modification. Not only does overall performance tend to improve or become more effective, but the underlying cognitive strategies change. Specifically, the formation of habits is thought to result from an incremental progression away from the use of flexible, attentive performance strategies to the engagement of inflexible, automatic strategies. This strategy transition is observed in various cognitive domains, e.g., spatial navigation (Hicks 1964; Packard and McGaugh 1996; Packard 1999; Schmitzer-Torbert 2007), instrumental and skill learning (Balleine and O'Doherty 2010; Derusso et al. 2010; De Kleine and Van der Lubbe 2011), and language interpretation and execution (Ullman 2004), and conserved across species (Schmitzer-Torbert 2007). Although the transition is robust, several factors internal and external to the subject alter the onset of automatic behaviors (Restle 1957; McDonald et al. 2004; Packard 2009). These factors seem to exert their effects by differentially engaging dissociable learning and memory systems which interact to recruit a distinct performance strategy at select time points during learning. Thus, an understanding of these interactions has important implications for decision-making at large.

Much of the current evidence for the nature of these interactions stems from studies of spatial navigation. In these studies, flexible/attentive strategies, termed place (locale) strategies, rely on calibrated spatial information associated with an expectancy

\section{${ }^{4}$ Corresponding author}

E-mail tdumas@gmu.edu

Article is online at http://www.learnmem.org/cgi/doi/10.1101//m.031336.113. of an outcome (Tolman 1948; O'Keefe and Nadel 1978), whereas inflexible/automatic strategies, termed response (taxon/praxic) strategies, rely on fixed motor sequences. During single-solution tasks, for which the use of spatial landmarks or a fixed motor sequence (but not both) are relevant to successful navigation, place and response navigational strategies show reciprocal interference and are thought to compete. For example, performance is diminished when an abundance of spatial cues (conducive to place learning) are presented during a task requiring response navigation (Packard 1987). In dual-solution tasks, for which the use of spatial landmarks and a fixed motor sequence are both suitable for successful performance, navigational strategies can work together to coordinate an appropriate action (Hamilton et al. 2004). Typical dual-solution designs include periodic probe trials to estimate the reliance on place and response navigation. Probe trials intermittently spaced across training have reliably demonstrated the strategy transition in spatial navigation, showing a greater reliance on place strategies early in training and response strategies after extensive repetition (Hicks 1964; Packard and McGaugh 1996; Packard 1999; Schmitzer-Torbert 2007).

Previous work has predominantly focused on strategy interactions during a single isolated decision, which precludes an

\footnotetext{
(C) 2013 Gardner et al. This article is distributed exclusively by Cold Spring Harbor Laboratory Press for the first 12 months after the fullissue publication date (see http://learnmem.cshlp.org/site/misc/terms. xhtml). After 12 months, it is available under a Creative Commons License (Attribution-NonCommercial 3.0 Unported), as described at http://creativecommons.org/licenses/by-nc/3.0/.
} 
understanding of strategy interplay during serial-choice learning. To investigate strategy interactions across sequential decisions, using spatial navigation in rodents as a model, we designed and built the Opposing Ts (OpT) maze, which permits experimental control over the strategies used on sequential intersections. We found that inclusion of a two-choice task that required attentive navigation, i.e., sustained use of spatial working memory (McDonald and White 1993; Devan et al. 2011), at the second choice prevented the transition to response navigation at the first choice. However, this effect was not observed in two-choice control tasks. Moreover, when secondary attentive training was initiated after extensive pretraining on a single dual-solution turn, its influence on primary strategy engagement was lost, i.e., fixed motor responses persisted indefinitely. These findings suggest that performance strategies interact across serial decisions and identify conditions that modulate the influence of subsequent choices on strategy recruitment.

\section{Results}

Rats were pseudo-randomly assigned to one of four tasks (Win-Shift, $n=16$; Win-Stay, $n=14$; Win-Win, $n=13$; and Plus, $n=20$ ), which differed in their cognitive requirement to find a food reward. For each task, rats underwent $5 \mathrm{wk}$ of daily training sessions administered on a sequential two-choice apparatus (the OpT maze) (Fig. 1). Win-Shift, Win-Stay, and Win-Win tasks utilized two consecutive choice points (primary and secondary) (Fig. 1C). On these three tasks, the primary choice invariably utilized a dualsolution turn, i.e., a turn that could be solved by reference memory engaging either place or response navigation. The learning demands on the secondary choice varied by task (Fig. 1D). The Win-Shift task required sustained attentive navigation on the second choice, i.e., flexible actions reliant on working memory for recent spatial exploration (e.g., McDonald and White 1993). This was accomplished by requiring a shift strategy from an immediately preceding run on which a random secondary arm was rewarded. In contrast, the secondary choice on the Win-Stay task (similar to the primary turn) could be solved by reference memory, engaging either place or response navigation. This was accomplished by invariably rewarding a secondary arm, found either by its fixed location or a fixed route. The Win-Win task demanded the same sensory-motor experiences as Win-Shift and Win-Stay tasks, but did not require secondary learning or memory, i.e., both secondary arms were rewarded. Between-task comparisons permitted isolation of the unique effects of secondary learning strategies on the primary place-to-response transition that occurs with overtraining. Inclusion of a dual-solution, single-choice task (Plus) provided a control condition to which the effects on primary strategy of secondary tasks could be compared more generally (Fig. 1E). An additional group of rats (P-WSh, $n=14$ ) was pretrained on the single-choice Plus task for $2 \mathrm{wk}$ prior to initiation of secondary Win-Shift training (see Materials and Methods for full details on each task).

\section{Primary choice accuracy and reward latency are equivalent between tasks}

On the primary choice, all rats reduced arm entry errors over the course of $6 \mathrm{~d}$ of training $\left(F_{(3.67,260.35)}=52.79, P<0.001\right)$, reaching $>90 \%$ accuracy (Fig. 2A; Supplemental Fig. 1A). While accuracy in movement toward the food reward was maintained $(>90 \%)$ with continued training, rats on the Win-Win task displayed an increased number of errors compared to rats on the Plus condition $(P<0.01)$. However, on average only a $3 \%$ performance decrement drove this effect. Reward latency also decreased with training $\left(F_{(4.40,312.30)}=42.80, P<0.001\right)$ in a similar fashion for each task (Fig. 2B; Supplemental Fig. 1B). Taken together, differences in secondary learning demands did not meaningfully alter performance on the primary choice.

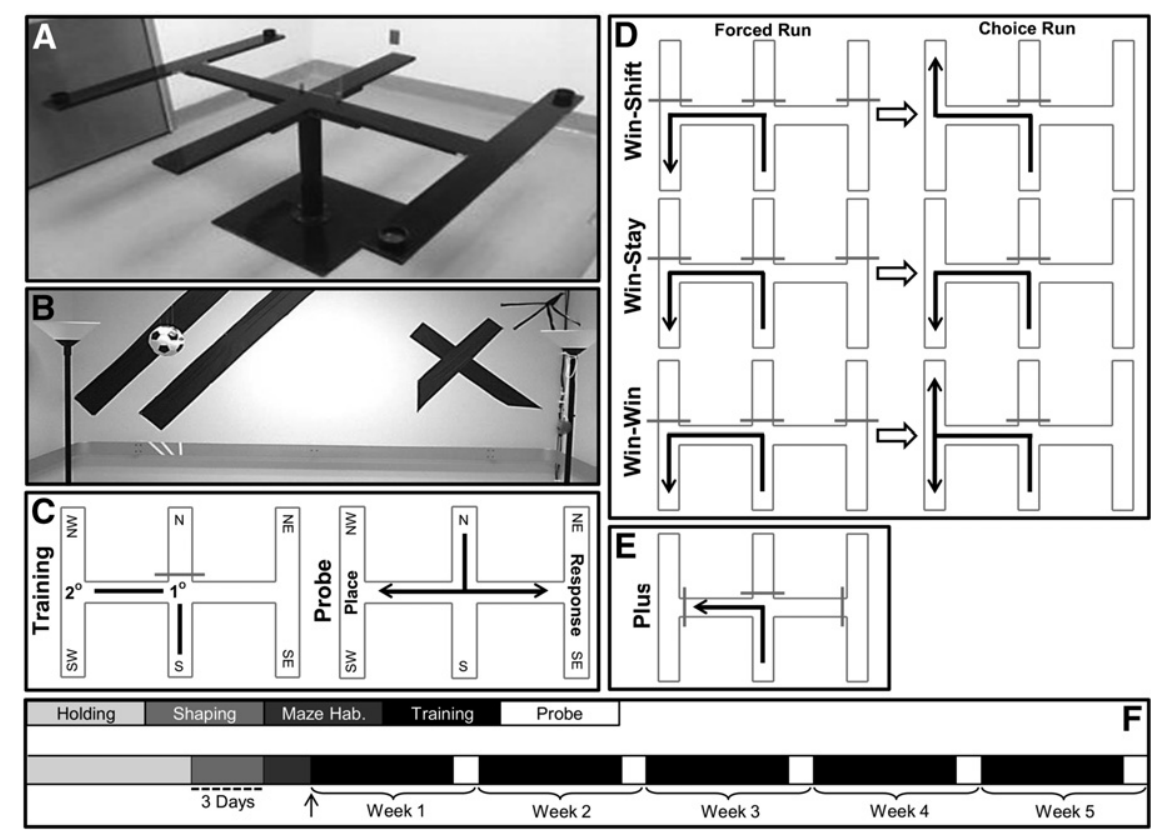

Figure 1. The OpT maze allows investigation of distinct navigational strategies across serial choice points. (A) Training was administered on the Opposing Ts (OpT) maze, which permitted sequential choice points (primary, $1^{\circ}$, and secondary, $2^{\circ}$, in $C$ ) that could vary in strategic demand. (B) The maze was positioned in a room with a moderately rich extra-maze environment. Each rat was assigned to one of four tasks (Win-Shift, Win-Stay, Win-Win, and Plus). (C) During training, rats were started from the south arm. Win-Shift, Win-Stay, and Win-Win tasks utilized primary and secondary choice points. The primary choice could be solved using either a place or a response strategy. However, the strategies needed to solve the secondary choice varied by task. $(D)$ Each trial was comprised of two paired runs: forced followed by choice. On the forced run, a blockade on the secondary choice (pseudo-randomly positioned for Win-Shift and Win-Win tasks, invariably positioned for the Win-Stay task) forced the animal to enter the open (rewarded) arm. On the choice run, no blockades were present on the secondary choice and a reward was positioned according to specific task demands. On the choice run, the Win-Shift task rewarded entry into the secondary arm blocked on the forced run (requiring spatial working memory). The Win-Stay task rewarded the same arm as on the forced run (solvable by reference memory). The Win-Win task rewarded both secondary arms (not requiring learning or memory). (E) The Plus task consisted of a single dual-solution turn on the primary choice point, and restricted access to secondary arms. On every seventh day after the start of training, a probe (five in total) was administered to identify strategy selection $(C, F)$. Arrows indicate the location of food rewards (Froot Loop cereal halves) $(D, E)$. See Supplemental Videos A, B for visual presentation of Plus and Win-Shift task training and probe runs. $(F)$ The experimental timeline shows the progression of each rat through the experiment prior to (i.e., Holding, Shaping, Maze Habituation) and following the onset of training (indicated by the arrow). 

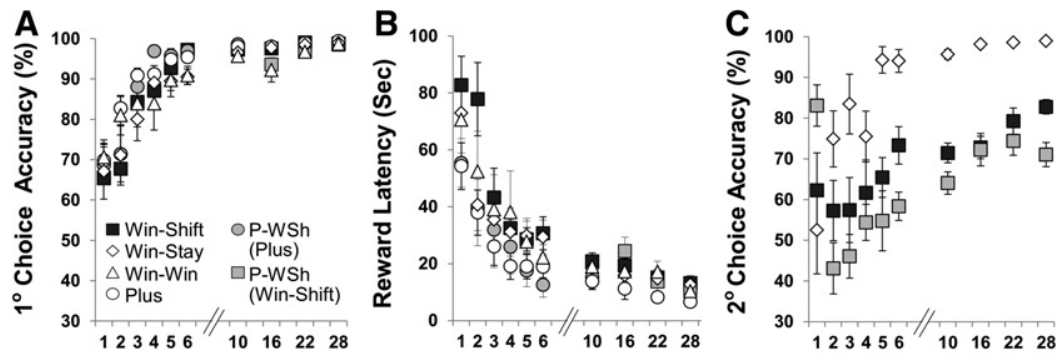

Days of Training

Figure 2. Rates of learning on primary and secondary choice points and reward latency by task. $(A)$ Task accuracy on the primary choice increased with training and reached asymptotic values after the first week. Across tasks, rats maintained $>90 \%$ accuracy on the primary choice with overtraining. (B) Reward latency was reduced throughout training with no task-specific effects. (C) Secondary task accuracy increased with training. The Win-Stay task was acquired more quickly than the Win-Shift task with (P-WSh) and without Plus pretraining. While Win-Stay rats reached asymptotic performance, Win-Shift performance continued to increase with extended training with similar learning rates in Win-Shift and P-WSh conditions. Despite an equivalent learning rate, Plus pretraining significantly increased the number of Win-Shift errors across training sessions. Data are shown as mean \pm SEM. After the first 6 $\mathrm{d}$ of training, values presented are averaged across each training week. The Win-Shift phase of the P-WSh group (P-WSh, Win-Shift) begins on training week three $(A, B)$. Rats per condition: Win-Shift, $n=16$; Win-Stay, $n=14$; Win-Win, $n=13$; Plus, $n=20$; P-WSh, $n=14$.

\section{Secondary choice accuracy differs according to task assignment}

All rats reduced secondary choice errors over the course of training $\left(F_{(11.66,431.45)}=6.87, P<0.0001\right)$. However, significant task differences in secondary accuracy were observed $\left(F_{(2,37)}=67.12, P<\right.$ 0.001). Win-Stay assigned rats quickly learned the secondary choice task during the first training week (>90\% accuracy), and maintained this level of accuracy during subsequent training sessions (Fig. 2C). Moreover, the learning rate demonstrated on the secondary choice mimicked that observed on the primary choice (cf. Win-Stay in Fig. 2A,C), suggesting they were learned in parallel. Rats also performed significantly above chance on the Win-Shift task, with (P-WSh reaching $\sim 74 \%$ accuracy, $P<0.0001$ ) or without (Win-Shift reaching $\sim 83 \%$ task accuracy, $P<0.0001$ ) Plus pretraining. At the same time, both conditions showed increased secondary errors compared to that observed in the Win-Stay condition $(P<0.05)$. Furthermore, Plus pretraining significantly increased the number of Win-Shift errors $(P<0.05)$. However, secondary Win-Shift accuracy in the P-WSh group, although reduced overall, increased with training at a rate similar to that observed in the Win-Shift group $(P>0.10)$ (Fig. 2C).

\section{Secondary task assignment differentially affects primary strategy use across training sessions}

The strategy used by a rat to navigate the primary choice was identified (probed) every seventh day following the onset of training by starting the rat from the opposite (north) arm to that during training (south) (Fig. 1C,F). On these probe runs, a place strategy was defined as entry into the side of the maze rewarded during training (indicating reliance on distal spatial cues), and a response strategy as use of the same body turn as rewarded during training (Fig. 1C; see Materials and Methods). After $6 \mathrm{~d}$ of training, each task produced a similar proportion of rats using a place strategy, i.e., $\sim 42 \%(P>0.10)$ (Fig. 3A; Supplemental Fig. 2). As expected, response strategies on the dual-solution Plus task increased significantly following continued training (e.g., from 55\% after $6 \mathrm{~d}$ of training to $90 \%$ after $30 \mathrm{~d} ; \chi^{2}(4)=9.94, P<0.05$ ) (e.g., Supplemental Video A), reflecting the transition from attentive to automatic performance. In contrast, secondary Win-Shift training maintained the number of rats using primary place strategies training was able to increase reliance on primary place strategies following the emergence of response navigation, a group of rats (P-WSh) was trained on the Plus task for 2 wk prior to the onset of Win-Shift training. As expected, 2 wk of Plus pretraining induced relative reliance on response strategies at the primary choice, a strategy profile consistent with that observed from rats assigned to the Plus task $(P>0.05)$ (Fig. 3A;
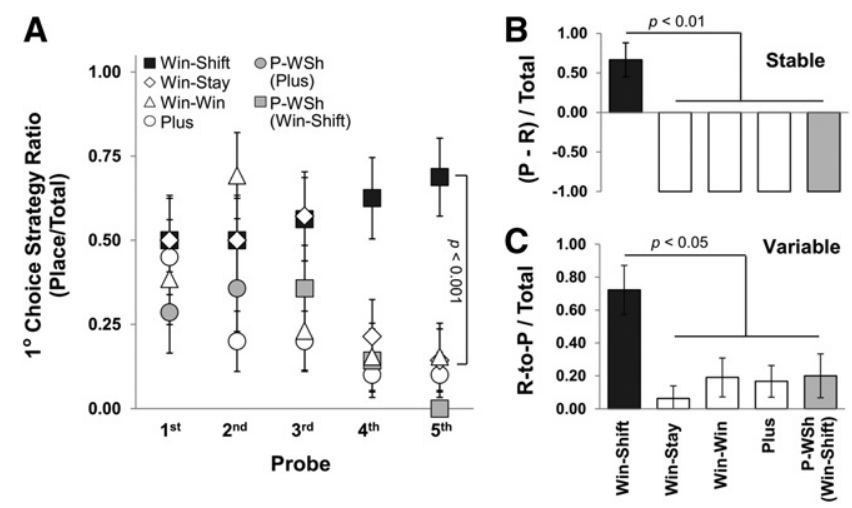

Figure 3. Win-Shift task assignment blocks the transition to response navigation on the primary choice. The incidence of primary response strategies increased with overtraining in all but the Win-Shift task. This is illustrated by plotting the primary choice strategy ratio (Place Rats Total Rats \pm SEM) for each task across probe runs ( $[A]$ : Win-Shift, $n=$ 16; Win-Stay, $n=14$; Win-Win, $n=13$; Plus, $n=20$; P-WSh, $n=14$ ). Strategy data from the Win-Shift phase of the P-WSh group (P-WSh, Win-Shift) begins with probe three. These data show that Win-Shift task assignment modifies primary strategy recruitment in favor of place navigation. In addition, Plus pretraining negated Win-Shift's influence over primary strategy. Rats were divided into stable place $(P)$, stable response $(R)$, variable place-to-response (P-to-R), and variable response-to-place (R-to-P) strategy profiles. The proportion of rats in each strategy profile is plotted for each task (stable rats: Win-Shift, $n=6$; Win-Stay, $n=4$; Win-Win, $n=2$; Plus, $n=5$; P-WSh, $n=5$; variable rats: Win-Shift, $n=$ 9; Win-Stay, $n=10$; Win-Win, $n=11$; Plus, $n=15$; P-WSh, $n=9$ ). Variable rats were weighted according to the extent of their transition (see Materials and Methods). The ratio of rats using stable $(B)$ to variable (C) strategies was equivalent between tasks; however, more place rats and more response-to-place rats were found in the Win-Shift task. Data compiled across probe runs from the P-WSh condition $(B, C)$ represent those collected during the Win-Shift training phase. 
Supplemental Fig. 2). However, Plus pretraining also blocked the effects of secondary Win-Shift training on strategy as shown by the maintained use of response navigation throughout the experiment. Consequently, like Plus, Win-Stay, and Win-Win tasks, after $30 \mathrm{~d}$ of training the P-WSh condition produced significantly fewer rats using a primary place strategy as compared to the Win-Shift task without Plus pretraining $(P<0.0001)$ (Fig. 3A; Supplemental Fig. 2). Moreover, this response dominant strategy profile of P-WSh rats after 3 wk of secondary Win-Shift training remained stable after an additional 2 wk of training (Supplemental Fig. 2), showing no effect of extended secondary spatial working memory training on primary strategy.

To better understand the task-specific aggregate strategy data across training sessions, individual rats were grouped into two broad strategy categories, "stable" and "variable" (Fig. 3B,C). Rats using a stable strategy invariably displayed the same strategy on each and every probe run (Fig. 3B). The remaining rats were grouped as having a variable strategy profile, and used each strategy at least once. Stable rats were subdivided according to place or response tendencies. Variable rats were subdivided according to the direction of their strategy transition across training sessions, i.e., from place-to-response or from response-to-place (Fig. 3C; see Materials and Methods). Each task produced an equivalent proportion of stable to variable rats $(P>0.10)$. However, the Win-Shift task produced a greater number of both stable rats using a place strategy $\left(\chi^{2}(4)=12.38, P<0.01\right)$ and variable rats showing a response-to-place strategy transition $\left(\chi^{2}(4)=9.81, P<0.05\right)$. In addition, within-task comparisons revealed five times the number of stable place (as compared to stable response) rats, and two times the number of variable response-to-place (as compared to variable place-to-response) rats in the Win-Shift group. These findings suggest that primary place navigation observed in rats assigned to the Win-Shift task (while maintained throughout training) is predominantly the cumulative result of rats utilizing a stable place strategy and those transitioning from a response to a place strategy. Thus, although the Win-Shift primary strategy ratio (place/total) on average remains close to chance levels (Fig. 3A; Supplemental Fig. 2), this result is not due to random arm entry on probe runs. Strategy subdivisions did not differentiate primary $(P>0.10)$ or secondary $(P>0.10)$ (Table 1$)$ choice accuracy or reward latency $(P>0.10)$, showing that task performance is robust to strategy engagement (Table 1). Altogether, these data suggest that secondary spatial working memory training maintains reliance on place strategies on a primary choice despite overtraining, but its effect is prevented by 2 wk of previous single-choice dualsolution training.

\section{Vicarious trial and error is selectively associated with place navigation}

Vicarious trial and error (VTE) is a term used to characterize back and forth movement at a choice point (Muenzinger and Gentry

Table 1. Strategy does not differentiate secondary choice accuracy

\begin{tabular}{lllllllll}
\hline Task & \multicolumn{1}{c}{$\mathbf{5} \mathbf{0}^{\mathbf{a}}$} & $\mathbf{4 , 1}$ & $\mathbf{3 , 2}$ & $\mathbf{2 , 3}$ & $\mathbf{1 , 4}$ & $\mathbf{0 , 5}$ & Total & $\boldsymbol{r}^{\mathbf{b}}$ \\
\hline Win-Stay & - & $95(2)$ & $97(3)$ & $93(3)$ & $94(-)$ & $95(1)$ & $95(2)$ & 0.07 \\
Win-Shift & $\mathbf{7 4}(10)$ & $80(2)$ & - & $73(6)$ & $73(7)$ & $77(7)$ & $74(7)$ & 0.07 \\
P-WSh & - & - & - & - & $66(8)$ & $70(-)$ & $68(8)$ & 0.24 \\
\hline
\end{tabular}

Secondary task accuracy (percent correct), shown as mean (standard deviation), is equivalent across strategy groupings.

a Strategy combinations (place, response) observed across the five probe runs administered during secondary task training.

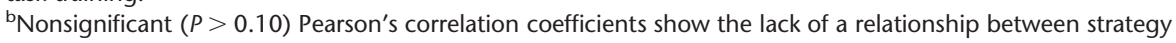
and accuracy within each secondary task.

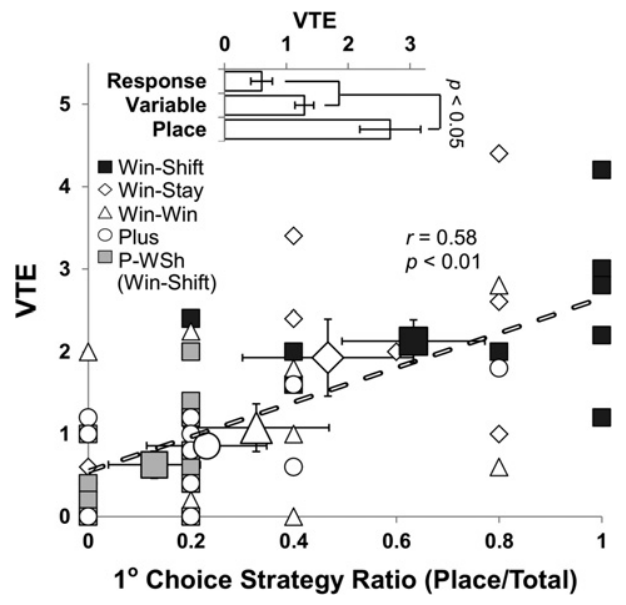

Figure 4. Vicarious trial and error is associated with place navigation and preserved during secondary Win-Shift training. Mean vicarious trial and error (VTE) was directly correlated with mean strategy $(r=0.58$, $P<0.01$ ), showing increased VTE from rats with relatively greater reliance on place navigation. Likewise, a greater number of VTE events were found in place compared to variable and response strategy groupings (mean \pm SEM, inset). Larger symbols reflect mean strategy and VTE ( \pm SEM) across rats according to task assignment, illustrating a between-task gradient in VTE and place-based navigation (ranked in decreasing order of mean VTE: Win-Shift, $n=12$; Win-Stay, $n=9$; Win-Win, $n=11$; Plus, $n=13$; P-WSh, $n=14)$. Data shown from the P-WSh condition represent those collected during the Win-Shift training phase.

1931; Muenzinger 1938; Tolman 1948). As VTE is thought to be involved in deliberative spatial navigation (Papale et al. 2012; van der Meer et al. 2012), we hypothesized that VTE would be selectively increased during place navigation and in rats assigned to the Win-Shift task. We defined VTE as the number of partial arm crosses at the primary choice point prior to arm entry and measured these values on probe runs. Such measurement revealed that the proportion of place strategies used by a rat was directly correlated with the amount of VTE $(r=0.58, P<0.0001)$ (Fig. 4), a trend mimicked when looking at individual strategy groupings $\left(F_{(3,50)}=7.54, P<0.01\right)$ (Fig. 4 , inset). Like strategy recruitment, the amount of VTE observed after $6 \mathrm{~d}$ of training was equivalent between tasks $(P>0.10)$. However, after $30 \mathrm{~d}$ of training, there was significantly more VTE observed in Win-Shift trained rats $(P<$ 0.05) (e.g., see Supplemental Videos A, B; cf. VTE during Plus to Win-Shift probe runs). Considering VTE on place and response runs separately for each task (as correlation analysis between VTE and strategy is confounded by task assignment), we found a robust increase ( $\sim 61 \%)$ in VTE during place as compared to response navigation, although considerable variability in the magnitude of this increase was observed across tasks (Win-Shift $=66 \%$, Win-Stay $=$ $150 \%$, Win-Win $=20 \%$, Plus $=20 \%$, P-WSh $=51 \%$ ). While the scoring method we used may not be sensitive to fine head movements and does not account for pausing (Hu et al. 1997), we also measured the duration of time rats spent in distinct segments of the maze on probe runs. The results from this analysis were consistent with the results obtained from tallies of partial arm crosses, showing that the mean time spent in the primary choice point was significantly greater on place $(10.58 \mathrm{sec})$ than on response runs $(5.76 \mathrm{sec}$, $\left.F_{(1,317)}=9.34, P<0.0001\right)$, an effect not observed on other maze segments, e.g., 
start or goal arms $(P>0.10)$. Together, these data reflect a robust correspondence between VTE and place navigation.

\section{Discussion}

This research set out to examine interactions between performance strategies during serial-choice learning. In particular, we investigated the effects of secondary tasks with specified cognitive requirements on the transition from attentive to automatic performance that occurs with repeated practice and experience. The findings reported here suggest the presence of a transfer effect of strategy during serial navigation, and outline conditions that modulate its occurrence. Specifically, we identified a strictly behavioral treatment that prevents, but does not reverse, the transition from the use of place (attentive) to response (automatic) strategies. Confirming previous research, we demonstrated that overtraining on a single-choice, dual-solution task (Plus) increased the incidence of response navigation (Fig. 3A; Supplemental Fig. 2; Hicks 1964; Packard and McGaugh 1996; Packard 1999). In contrast, we found that a two-choice task that required attentive performance (Win-Shift, spatial working memory) at the second intersection completely blocked the transition from place-to-response navigation at the first (primary) (Fig. 3A; Supplemental Fig. 2). In addition, control two-choice tasks that could be solved using reference memory and/or response navigation, but that overlapped considerably in many behavioral aspects (Win-Stay, Win-Win), delayed but did not prevent the onset of response strategies with overtraining. Together, these results suggest that while secondary reference memory and/or additional sensory-motor experiences may delay the strategy transition, secondary spatial working memory training is able to preserve the reliance on place performance at a primary choice.

The extent and rate of secondary learning was decreased in the Win-Shift task compared to control tasks (Fig. 2C). Therefore, we cannot eliminate secondary learning and task difficulty as factors influencing primary strategy recruitment. Indeed, uncertainty has been shown to modify the use of performance strategies (Derusso et al. 2010; Sullivan et al. 2012). However, it appears that uncertainty, learning phase, and/or task difficulty on the secondary choice are not principal factors driving the effects on primary strategy observed here. In particular, although rats in the Win-Shift condition did not reach asymptotic secondary performance, they significantly increased accuracy across training sessions (Fig. 2C). Moreover, secondary task accuracy did not predict primary strategy groupings both in the aggregate (cf. Win-Shift in Figs. 2C, 3A; Supplemental Fig. 2) and individual data (Table 1). In fact, while there was a nonsignificant increase in primary place strategies with continued Win-Shift training, a significant transition to response navigation was observed in control tasks, although secondary performance was increased with training on all tasks.

Similarly, our experimental design did not isolate the effects of working memory for spatial locations as opposed to working memory for sequences of actions or turns. For example, although the Win-Shift task requires an animal to attend to relevant task information (Zola-Morgan and Squire 1985), and recruits the same neural systems implicated in place/spatial strategies (Packard et al. 1989; McDonald and White 1993; Packard and McGaugh 1996; Packard 1999; Devan et al. 2011), it is possible that some Win-Shift animals strictly relied on working memory for recently used routes (as opposed to spatial landmarks) to locate the goal. Thus, it would be interesting to test the distinct contributions of secondary spatial memory and working memory (in addition to task difficulty) on the regulation of primary strategy recruitment. A design that includes additional conditions, e.g., fixed, higher- order serial tasks that vary in difficulty, and a cued nonspatial working memory task (McDonald and White 1993), would help disentangle these factors to better understand their individual influences on strategy engagement during serial decision-making.

The interaction between attentive and automatic performance strategies across decision points observed here complements human studies showing transfer effects of working memory training to unpracticed tasks that utilize similar brain structures and cognitive processes (Klingberg 2010; Brehmer et al. 2012). Moreover, in agreement with working memory experiments that measure capacity (Huang-Pollock and Karalunas 2010), these results suggest that working memory demand is inversely associated with implicit skill acquisition or automatic performance. To determine if secondary Win-Shift training directly inhibits primary response engagement and/or directly facilitates primary place engagement will require further investigation.

VTE is a marker of deliberative spatial processing in the rat. We found VTE to selectively co-occur with place-dependent learning strategies on a dual-solution turn, a finding consistent with Schmidt et al. (2013) showing VTE is increased during explicit place compared to response training. Additionally, VTE behaviors were strongly associated with the prevention of the placeto-response transition by secondary Win-Shift training. As the Win-Shift task requires ongoing updating and application of recent spatial information, these results support the hypothesis that VTE is more akin to an active search mechanism (Johnson et al. 2012; Papale et al. 2012), rather than a passive exploratory behavior.

Together, these findings support the notion that secondary working memory training has potential value to increase attentive performance on targeted tasks and skills despite extensive practice and experience (e.g., Di Nocera et al. 2006; Youmans and Ohlsson 2008; Hagewoud et al. 2010; Friederich and Herzog 2011; Gillan et al. 2011; He et al. 2011; Reichenbach et al. 2011; Hogarth et al. 2013). Consistent with this hypothesis, delay discounting has been shown to improve both with working memory training (Bickel et al. 2011) and instruction that shifts attention to focus on later rather than immediate rewards (Radu et al. 2011).

However, our data also suggest that automatic performance resulting from repeated practice may be less responsive to such treatment. The increase in rats transitioning from a response to a place strategy in the Win-Shift task presents the possibility that secondary working memory training is able to increase the use of attentive strategies after sustained reliance on fixed motor behaviors. However, we did not see this effect after 2 wk of Plus pretraining, which produced a relative reliance on response navigation (Fig. 3A; Supplemental Fig. 2). These discrepant findings are potentially explained by methodological differences between the Win-Shift task with (P-WSh) and without Plus pretraining. Specifically, the Plus task rewards the primary turn at the end of the east or west arm. In contrast, the Win-Shift task demands primary and secondary choices, but grants a single reward at the end of the "correct" secondary arm (see Materials and Methods). Therefore, prior learning in the Plus configuration could elicit compartmentalization of the primary and secondary choice points into discrete tasks that rely on discrete strategies, an effect that persists across Win-Shift training sessions. Alternatively, as rats pretrained on the Plus task reached asymptotic performance and predominantly showed response strategies on the primary choice after 2 wk of training, the influence of subsequent Win-Shift training on primary strategy may have been blocked by previous training and/or response interference. This notion suggests there may be a sensitive period during which secondary Win-Shift training can act upon primary strategy recruitment.

In accordance with this latter explanation, compared to Win-Shift success when training began at the onset of the 
experiment, secondary Win-Shift accuracy was decreased following Plus pretraining, demonstrating some degree of performance interference. This result is consistent with findings from a dualsolution task that showed reduced performance during subsequent strategy reversal training (on the same choice point) after overtraining (Hicks 1964). Extending this research, our findings suggest that strategy interference may occur across choice points, during which the use of previously utilized strategy/choice-point combinations (e.g., the use of an automatic strategy on an initial decision) impairs acquisition of a subsequent task requiring the alternative strategy (e.g., a subsequent attentive task). Further testing will help determine the degree to which primary strategy, as opposed to nonspecific learning, influences secondary singlesolution learning. At large, our data suggest that administration of a secondary working memory task may preserve attentive decision-making, and is most effective when administered from the onset of task acquisition. That is, once a task is overtrained, even prolonged secondary working memory training may not be an effective means to modify the underlying performance strategy. The initiation of secondary Win-Shift training only varied across two time points, i.e., at the start of the experiment or following 2 wk of Plus pretraining. Therefore, it is unclear if the effects of secondary spatial working memory training on primary strategy are "all or none" or graded. For example, it is possible that as behaviors become more rigid with increased training periods, the efficacy of working memory training is gradually reduced. Identifying the nature of these effects will help resolve whether secondary working memory training may be effective at increasing the use of attentive performance strategies in specified overtrained behaviors. Similarly, this research did not test whether response navigation, as determined on probe runs, was habitual. However, previous research using a modified dual-solution procedure paired with reward devaluation showed that after $10 \mathrm{~d}$ of overtraining (40 runs/d) performance was habitual (Smith et al. 2012). Therefore, the results reported here on the malleability of the transition to response navigation may be applicable to the transition to habitual behaviors with repeated performance.

In rodents, a robust double dissociation is observed between a hippocampal-mediated place strategy and a dorsolateral striatalmediated response strategy (Packard et al. 1989; Packard and McGaugh 1996; Packard 1999; Yin and Knowlton 2004); with similar dissociations noted in humans (Corkin 1968; Knowlton et al. 1996; Hartley et al. 2003; Squire 2004; Bohbot et al. 2007; Balleine and O'Doherty 2010; Banner et al. 2011; Wit et al. 2012). Moreover, several factors that modulate strategy recruitment are shown to act differentially on neural structures implicated in place and response navigation (Packard 1987, 2009; McDonald et al. 2004). In light of these findings, and those showing that successful Win-Shift performance and VTE are dependent upon place-based brain regions (McDonald and White 1993; Hu and Amsel 1995; Hu et al. 1997, 2006; Devan et al. 2011), it is tempting to discuss the results of this research in terms of task-specific engagement of place and response neural circuits. We put forth a hypothesis that maintenance of a place strategy on the primary choice point (despite overtraining) during Win-Shift training is due to specified secondary recruitment of the hippocampus and supporting place-based neural structures (e.g., dorsomedial striatum; Yin and Knowlton 2004). As Win-Stay and Win-Win tasks produce a comparable, albeit transient, effect on strategy, the simplest explanation would assert that these tasks also exert their effects through a time-limited hippocampal-mediated mechanism. In support of this idea, learning of the second turn of a two-turn response-based task was disrupted in a transgenic line of mice lacking $\mathrm{N}$-methyl-D-aspartate receptor GluN1 subunits in CA1 of the hippocampus (Rondi-Reig et al. 2006), a finding consistent with the role of hippocampus in sequence learning and memory
(Kesner et al. 2002). This two-turn response-based task was administered for $\sim 2 \mathrm{wk}$. Therefore, it is unclear if a more extensive training period would permit secondary performance to be supplemented by an alternative neural mechanism and learning strategy. Our findings that show an increase in primary response navigation with extended training on Win-Stay and Win-Win tasks, however, support this notion.

Consistent with the hypothesis that the effects of secondary Win-Shift training on primary strategy reliance are mediated by the hippocampus, spatial training has been shown to increase gray matter selectively in the hippocampus (Maguire et al. 2006; Lerch et al. 2011), a factor directly associated with increased reliance on place strategies (Bohbot et al. 2007). Further work will need to clarify the degree to which secondary training can influence strategy reliance in subjects with a response predisposition, e.g., as a result of aging (Bohbot et al. 2012), neuroanatomical variability (Bohbot et al. 2007; Wit et al. 2012), genetic polymorphism (Banner et al. 2011), stress (Packard 2009; Schwabe et al. 2010), and/or sleep deprivation (Hagewoud et al. 2010), rather than repeated practice and experience. Nonetheless, this research opens a promising line of future investigation. We emphasize, however, that even slight alterations to training designs can significantly modify the neural structures supporting a particular task. Therefore, behavioral results obtained using the OpT maze in conjunction with measures of neural activity, plasticity, and causality will help further our understanding of the neural regulation of and the precise relationship between attentive and automatic memory systems.

\section{Materials and Methods}

\section{Animals}

Male Long-Evans Hooded rats (Charles River Laboratories, Wilmington, MA), 275-500 g, were pseudo-randomly assigned to one of four tasks (Win-Shift, $n=16$; Win-Stay, $n=14$; Win-Win, $n=13$; and Plus, $n=20$ ) (Fig. 1D,E). In addition, a separate group of rats (P-WSh, $n=14$ ) was given 2 wk of pretraining on the Plus task followed by training on the Win-Shift task. All methods were in accordance with the National Institutes of Health Guide for the Care and Use of Laboratory Animals and were approved by the George Mason University Institutional Animal Care and Use Committee.

\section{Apparatus}

Tasks were administered on the OpT maze (Opposing Ts) (Fig. 1), an adaptation of the Plus maze (e.g., Tolman et al. 1946; see also Pol-Bodetto et al. 2011) with secondary arms attached to and bisected by the ends of the east and west arm segments of the "plus." Thus, it is comprised of four primary maze segments, i.e., north, east, south, west, and four secondary maze segments, i.e., northeast, northwest, southeast, and southwest (Fig. 1C), each measuring 23.5 in (length) $\times 4.5$ in (width) $\times 0.75$ in (height) built off square choice points measuring 4.5 in (length) $\times 0.75$ in (height). Each arm segment permits attachment of a removable blockade (Plexiglas) and an opaque, circular food (reward) cup measuring 1.25 in (height) and 2.5 in (diameter). The maze is constructed from pine boards painted flat black and coated with a clear lacquer. It swivels $360^{\circ}$ on its cylindrical base which elevates the maze $28 \mathrm{in}$. The testing room was environmentally enriched (Fig. 1B) with three-dimensional objects and geometric shapes hanging on the walls and ceiling. Furthermore, lighting was provided by four floor lamps placed in the northeast, northwest, southeast, and southwest corners of the room, each using 30-W bulbs.

\section{Habituation}

Upon import to our animal facility, rats were housed (2-3 per cage) for a minimum of $7 \mathrm{~d}$. Rats were then individually housed 
and brought to $85 \%$ of their free-feeding weight through caloric restriction over an additional $7 \mathrm{~d}$. Concurrent with caloric restriction, rats were handled 5-min daily ("Holding" in Fig. 1F; see Packard and McGaugh 1996). Following this period, rats were habituated to Froot Loop (FL) cereal (Kellogg) by placing three FL halves in their home cage. Rats were shaped daily to take and consume a FL half from a reward cup positioned at the end of a rectangular table in a room distinct from that used for training ("Shaping" in Fig. 1F). After consuming the FL half for at least three consecutive days and under $180 \mathrm{sec}$ on the final $2 \mathrm{~d}$, rats were habituated to the OpT maze ("Maze Hab." in Fig. 1F). On each day, for $2 \mathrm{~d}$, maze habituation provided 5 min of maze exploration starting the rat from the south arm with the north arm blocked (e.g., Packard and McGaugh 1996). During maze habituation, single FL halves were placed in all reward cups, with one cup positioned at each possible reward site (at the ends of the east and west arm segments for the Plus task, and at the ends of the four secondary arm segments for Win-Shift, Win-Stay, and Win-Win tasks). The experimenter recorded the rat's first arm entry. The side recorded on day 2 of maze habituation was identified as the rat's arm/turn preference.

\section{Tasks}

After maze habituation, training began and proceeded daily for 5 wk (except for every seventh day when a strategy probe was administered) (Fig. 1F). During training, the north arm was blocked and the animal was started from the south arm (Fig. 1C). Twelve runs were given daily with a 3-min run maximum and 30-sec inter-run interval during which the rat was kept in the holding cage behind the south arm. Throughout training, the animal was rewarded to the side of the maze (east or west) opposite its preference (e.g., Tolman et al. 1946) identified during day 2 of maze habituation. The reward consisted of a single FL half placed in the reward cup positioned at the end of the appropriate arm segment according to task-specific training rules. During days 1 and 2 of training, the rat could retrace its steps to find the goal after entering a nonrewarded arm (e.g., Ritchie et al. 1950). With the exception of the P-WSh condition, for which this self-correction method was also applied to the secondary arms for the first $2 \mathrm{~d}$ of Win-Shift training, on day 3 and onward, after entering a nonrewarded arm, the rat was removed from the maze. Between runs, the maze was pseudo-randomly rotated $180^{\circ}$ and wiped down with water to minimize use of intra-maze cues. On the first two runs of the first day of training, a trail of four FL halves led the rat from the choice point to the baited reward cup. Arm entries and reward latency were documented by the experimenter with the aid of a silent-operation stopwatch.

Win-Shift, Win-Stay, and Win-Win tasks (Fig. 1D) included two consecutive choice points (primary and secondary) (Fig. 1C). The primary choice could be learned using either a place or response strategy and rely on reference memory. This primary turn was equivalent to that in the Plus task. However, to retrieve the food reward, each rat was required to navigate the secondary arms. Reward cups were placed at the end of each secondary arm. Each trial (six daily) consisted of a pair of runs (e.g., Dumas et al. 2004). One of the secondary arms was blocked on the first run leading the rat to find a reward on the open arm (forced run). On the second run, neither arm was blocked, requiring a choice to be made between the two open arms (choice run).

The learning demands of the secondary choice varied according to the assigned task. On the choice run, the Win-Shift task (e.g., McDonald and White 1993) rewarded entry into the previously blocked arm (assigned pseudo-randomly), requiring working memory. In contrast, the Win-Win task rewarded entry into either arm and thus did not require secondary learning. For the Win-Stay task, on forced runs, the blocked arm was invariably positioned opposing the start arm, and the same previously rewarded arm (on the forced run) was again rewarded on choice runs. Thus the Win-Stay task, by design, was a serial dual-solution task and could be solved by reference memory.

The Plus task (Fig. 1E) consisted of a single (primary) choice for which rats were rewarded when making a consistent turn from the south starting position (constants throughout training [Ritchie et al. 1950; Hicks 1964]). Thus, like the primary choice on each secondary task, the Plus task could be learned using longterm memory engaging either a place or response strategy. Entry into secondary arms was restricted by Plexiglas blockades at the ends of the east and west arms. Reward cups were placed at the ends of the east and west arms in front of the blockades.

To assess the strategies used on the primary choice across training sessions, on every seventh day a probe consisting of a single run was administered, starting the animal from the arm opposite to the training start arm (Fig. 1C,F; Hicks 1964; Packard and McGaugh 1996; Packard 1999). A place strategy was identified if the rat entered the same arm rewarded during training. A response strategy was recorded if the rat made the same turn rewarded during training (Fig. 1C; see Supplemental Videos A, B for examples of Plus and Win-Shift training and probe runs). Five total probes were administered for Win-Shift, Win-Stay, Win-Win, and Plus groups (Fig. 1F). Seven total probes were given in the P-WSh condition, two during Plus pretraining and five during Win-Shift training.

\section{Data analysis}

Maze habituation, training, and probe sessions were videotaped for subsequent analysis. During maze habituation, training, and probe runs, an arm entry was defined by the full body of the rat excluding the tail. Rats were excluded from analysis if $<75 \%$ accurate on the primary choice or displaying a reward latency mean $>2$ min on days prior to probe runs. This procedure excluded five rats ( $6 \%$ of total).

As rats were prevented from self-correcting after the initial 2-d interval, task accuracy on the secondary choice for Win-Shift, Win-Stay, and P-WSh conditions was calculated only if a rat was successful on the primary choice during both forced and choice runs. This procedure permitted orthogonal analyses of primary and secondary choice accuracy. Since the Win-Win task rewarded both secondary arms on choice runs, by design, rats in this condition could not make an incorrect choice and were thus excluded from secondary choice accuracy analyses.

Rats using a "stable" strategy invariably displayed the same strategy on each probe run. The remaining rats were grouped as having "variable" strategy use. Stable rats were subdivided according to place or response biases. Variable rats were subdivided according to the direction of their strategy transition, i.e., from place-to-response or from response-to-place. For each variable rat, this designation was determined by dummy coding place (1) and response (0) strategies and calculating the slope of the line of best fit across probes one through five. The sign of this line determined each variable rat's grouping; a negative slope indicated a transition from place-to-response navigation and a positive slope indicated a transition from response-to-place navigation. In the P-WSh condition, the line of best fit was calculated from the five probe runs administered during the Win-Shift phase of training (data were equivalent when accounting for all probes given both during Plus pretraining and Win-Shift training phases). For analysis, variable data (Fig. 3C) were weighted by the absolute value of the slope of the line of best fit. This approach provided greater influence to those rats with more robust transitions (data were equivalent when using unweighted counts of variable transitions).

VTE was quantified on the primary choice point of probe runs using experimenter-scored analysis of recorded videos. Similar to previous studies (e.g., Hu and Amsel 1995) measuring VTE by the number of head orientations to visual stimuli prior to action selection, we defined VTE as the number of unique partial head/body entries into the open arms surrounding the primary choice point prior to full body entry into either the east or west arm. This value was tallied for each probe session for each rat. For example, Supplemental Video A (early training probe) shows a sequence of partial arm crosses prior to west arm entry with the full body: (1) two paws in the east arm, (2) two paws in the west arm, and (3) two paws in the north arm. The VTE score for this probe run is three. If no such partial crosses were made, the resulting 
score is zero (e.g., Supplemental Video A, extended training probe). The duration of time spent in distinct portions of the maze on probe runs was also scored from videos by an experimenter with the aid of a stopwatch. Three regions of interest were applied to segment the maze: the starting arm, the primary choice point (defined by the central square), and the east and west arm segments. A subset of video files ( $22 \%$ in total) distributed across Win-Shift, Win-Stay, Win-Win, and Plus tasks was corrupted during back-up. Thus, not all probe runs were analyzed for VTE events or exploration of maze segments. However, complete sets of intact video files (i.e., video of all probe runs for a given rat) from animals representative of aggregate data in each task (Win-Shift, $n=12$; Win-Stay, $n=9$; Win-Win, $n=11$; Plus, $n=13$; P-WSh, $n=14$ ) permitted unbiased statistical comparison of VTE and arm segment exploration. In addition to arm entry designations on probe runs, all findings reliant on experimenter-scored video analysis were corroborated through further video analysis by researchers blind to secondary task assignment and research hypotheses.

Data compiled across probe runs from the P-WSh condition (i.e., Figs. 3B,C, 4) represent those collected during the WinShift training phase (weeks 3-7). Unless otherwise noted, data are presented as mean \pm standard error of the mean. A mixed model ANOVA was utilized to assess task, training duration, and strategy-grouping effects on primary and secondary choice accuracy, reward latency, and VTE. Single-sample $t$-tests were run to determine if secondary Win-Shift accuracy was above chance levels. Fisher's exact test, the $\chi^{2}$ test for homogeneity, Cochran's $Q$ test, and a mixed model logistical regression with binary variables, a procedure fit with the generalized estimating equation, were utilized to compare strategy use across tasks and training duration. Pearson's $r$ was calculated to assess correlation between strategy and secondary choice accuracy, and strategy and VTE. Statistical significance was interpreted using the criterion of $P<0.05$. All post-hoc tests were corrected for type- 1 error inflation using the Bonferroni technique. The Greenhouse-Geisser correction was applied to violations of sphericity (for full details on these statistical tests, see Davis 2002; Sheskin 2011).

\section{Acknowledgments}

We thank Asish Gulati, Iris Talebi, Mai-Phuong Hoang, Christopher Rudnicky, and Christina Pehlivanis for their help conducting behavioral experiments and with data analysis. This research was funded by the Office of Naval Research, ONR\#000141010198; the Air Force Office of Scientific Research (AFOSR), Award No. FA9550-10-1-0385; and the Krasnow Institute for Advanced Study.

\section{References}

Balleine BW, O'Doherty JP. 2010. Human and rodent homologies in action control: Corticostriatal determinants of goal-directed and habitual action. Neuropsychopharmacology 35: 48-69.

Banner H, Bhat V, Etchamendy N, Joober R, Bohbot VD. 2011. The brain-derived neurotrophic factor Val66Met polymorphism is associated with reduced functional magnetic resonance imaging activity in the hippocampus and increased use of caudate nucleus-dependent strategies in a human virtual navigation task. Eur J Neurosci 33: 968-977.

Bickel WK, Yi R, Landes RD, Hill PF, Baxter C. 2011. Remember the future: Working memory training decreases delay discounting among stimulant addicts. Biol Psychiatry 69: 260-265.

Bohbot VD, Lerch J, Thorndycraft B, Iaria G, Zijdenbos AP. 2007. Gray matter differences correlate with spontaneous strategies in a human virtual navigation task. J Neurosci 27: 10078-10083.

Bohbot VD, McKenzie S, Konishi K, Fouquet C, Kurdi V, Schachar R, Boivin M, Robaey P. 2012. Virtual navigation strategies from childhood to senescence: Evidence for changes across the life span. Front Aging Neurosci 4: 28

Brehmer Y, Westerberg H, Bäckman L. 2012. Working-memory training in younger and older adults: Training gains, transfer, and maintenance. Front Hum Neurosci 6: 63.

Corkin S. 1968. Acquisition of motor skill after bilateral medial temporal-lobe excision. Neuropsychologia 6: 255-265.
Davis CS. 2002. Statistical methods for the analysis of repeated measurements. Springer, New York.

De Kleine E, Van der Lubbe RHJ. 2011. Decreased load on general motor preparation and visual-working memory while preparing familiar as compared to unfamiliar movement sequences. Brain Cogn 75: $126-134$.

Derusso AL, Fan D, Gupta J, Shelest O, Costa RM, Yin HH. 2010. Instrumental uncertainty as a determinant of behavior under interval schedules of reinforcement. Front Integr Neurosci 4: 17.

Devan BD, Hong NS, McDonald RJ. 2011. Parallel associative processing in the dorsal striatum: Segregation of stimulus-response and cognitive control subregions. Neurobiol Learn Mem 96: 95-120.

Di Nocera F, Fabrizi R, Terenzi M, Ferlazzo F. 2006. Procedural errors in air traffic control: Effects of traffic density, expertise, and automation. Aviat Space Environ Med 77: 639-643.

Dumas TC, Powers EC, Tarapore PE, Sapolsky RM. 2004. Overexpression of calbindin D28k in dentate gyrus granule cells alters mossy fiber presynaptic function and impairs hippocampal-dependent memory. Hippocampus 14: 701-709.

Friederich H-C, Herzog W. 2011. Cognitive-behavioral flexibility in anorexia nervosa. Curr Top Behav Neurosci 6: 111-123.

Gillan CM, Papmeyer M, Morein-Zamir S, Sahakian BJ, Fineberg NA, Robbins TW, de Wit S. 2011. Disruption in the balance between goal-directed behavior and habit learning in obsessive-compulsive disorder. Am J Psychiatry 168: 718-726.

Hagewoud R, Havekes R, Tiba PA, Novati A, Hogenelst K, Weinreder P, Van der Zee EA, Meerlo P. 2010. Coping with sleep deprivation: Shifts in regional brain activity and learning strategy. Sleep 33: $1465-1473$.

Hamilton DA, Rosenfelt CS, Whishaw IQ. 2004. Sequential control of navigation by locale and taxon cues in the Morris water task. Behav Brain Res 154: 385-397.

Hartley T, Maguire EA, Spiers HJ, Burgess N. 2003. The well-worn route and the path less traveled: Distinct neural bases of route following and wayfinding in humans. Neuron 37: 877-888.

He J, Becic E, Lee Y-C, McCarley JS. 2011. Mind wandering behind the wheel: Performance and oculomotor correlates. Hum Factors 53: 13-21.

Hicks LH. 1964. Effects of overtraining on acquisition and reversal of place and response learning. Psychol Rep 15: 459-462.

Hogarth L, Balleine BW, Corbit LH, Killcross S. 2013. Associative learning mechanisms underpinning the transition from recreational drug use to addiction. Ann N Y Acad Sci 1282: 12-24.

$\mathrm{Hu} \mathrm{D}$, Amsel A. 1995. A simple test of the vicarious trial-and-error hypothesis of hippocampal function. Proc Natl Acad Sci 92: 5506-5509.

Hu D, Griesbach G, Amsel A. 1997. Development of vicarious trial-and-error behavior in odor discrimination learning in the rat: Relation to hippocampal function? Behav Brain Res 86: 67-70.

Hu D, Xu X, Gonzalez-Lima F. 2006. Vicarious trial-and-error behavior and hippocampal cytochrome oxidase activity during Y-maze discrimination learning in the rat. Int J Neurosci 116: 265-280.

Huang-Pollock CL, Karalunas SL. 2010. Working memory demands impair skill acquisition in children with ADHD. J Abnorm Psychol 119: $174-185$

Johnson A, Varberg Z, Benhardus J, Maahs A, Schrater P. 2012. The hippocampus and exploration: Dynamically evolving behavior and neural representations. Front Hum Neurosci 6: 216.

Kesner RP, Gilbert PE, Barua LA. 2002. The role of the hippocampus in memory for the temporal order of a sequence of odors. Behav Neurosci 116: $286-290$.

Klingberg T. 2010. Training and plasticity of working memory. Trends Cogn Sci (Regul Ed) 14: 317-324.

Knowlton BJ, Mangels JA, Squire LR. 1996. A neostriatal habit learning system in humans. Science 273: 1399-1402.

Lerch JP, Yiu AP, Martinez-Canabal A, Pekar T, Bohbot VD, Frankland PW, Henkelman RM, Josselyn SA, Sled JG. 2011. Maze training in mice induces MRI-detectable brain shape changes specific to the type of learning. Neuroimage 54: 2086-2095.

Maguire EA, Woollett K, Spiers HJ. 2006. London taxi drivers and bus drivers: A structural MRI and neuropsychological analysis. Hippocampus 16: $1091-1101$.

McDonald RJ, White NM. 1993. A triple dissociation of memory systems: Hippocampus, amygdala, and dorsal striatum. Behav Neurosci 107: $3-22$.

McDonald RJ, Devan BD, Hong NS. 2004. Multiple memory systems: The power of interactions. Neurobiol Learn Mem 82: 333-346.

Muenzinger KF. 1938. Vicarious trial and error at a point of choice: I. A general survey of its relation to learning efficiency. Pedagog Semin J Genet Psychol 53: 75-86.

Muenzinger FK, Gentry E. 1931. Tone discrimination in white rats. J Comp Psychol 12: 195-206.

O'Keefe J, Nadel L. 1978. The hippocampus as a cognitive map. Oxford University Press, Oxford, UK. 
Packard MG. 1987. Differential roles of hippocampus and caudate nucleus in memory: selective mediation of "cognitive" and "associative" learning. McGill University, Montreal, Canada.

Packard MG. 1999. Glutamate infused posttraining into the hippocampus or caudate-putamen differentially strengthens place and response learning. Proc Natl Acad Sci 96: 12881.

Packard MG. 2009. Exhumed from thought: Basal ganglia and response learning in the plus-maze. Behav Brain Res 199: 24-31.

Packard MG, McGaugh JL. 1996. Inactivation of hippocampus or caudate nucleus with lidocaine differentially affects expression of place and response learning. Neurobiol Learn Mem 65: 65-72.

Packard MG, Hirsh R, White NM. 1989. Differential effects of fornix and caudate nucleus lesions on two radial maze tasks: Evidence for multiple memory systems. J Neurosci 9: 1465-1472.

Papale AE, Stott JJ, Powell NJ, Regier PS, Redish AD. 2012. Interactions between deliberation and delay-discounting in rats. Cogn Affect Behav Neurosci 12: 513-526.

Pol-Bodetto S, Jeltsch-David H, Lecourtier L, Rusnac N, Mam-Lam-Fook C, Cosquer B, Geiger K, Cassel J-C. 2011. The double-H maze test, a novel, simple, water-escape memory task: Acquisition, recall of recent and remote memory, and effects of systemic muscarinic or NMDA receptor blockade during training. Behav Brain Res 218: 138-151.

Radu PT, Yi R, Bickel WK, Gross JJ, McClure SM. 2011. A mechanism for reducing delay discounting by altering temporal attention. J Exp Anal Behav 96: 363-385.

Reichenbach J, Onnasch L, Manzey D. 2011. Human performance consequences of automated decision aids in states of sleep loss. Hum Factors 53: 717-728.

Restle F. 1957. Discrimination of cues in mazes: A resolution of the place-vs.-response question. Psychol Rev 64: 217-228.

Ritchie BF, Aeschliman B, Peirce P. 1950. Studies in spatial learning. J Comp Physiol Psychol 43: 73-85.

Rondi-Reig L, Petit GH, Tobin C, Tonegawa S, Mariani J, Berthoz A. 2006. Impaired sequential egocentric and allocentric memories in forebrain-specific-NMDA receptor knock-out mice during a new task dissociating strategies of navigation. J Neurosci 26: 4071-4081.

Schmidt B, Papale A, Redish AD, Markus EJ. 2013. Conflict between place and response navigation strategies: Effects on vicarious trial and error (VTE) behaviors. Learn Mem 20: 130-138.
Schmitzer-Torbert N. 2007. Place and response learning in human virtual navigation: Behavioral measures and gender differences. Behav Neurosci 121: $277-290$.

Schwabe L, Schächinger H, de Kloet ER, Oitzl MS. 2010. Corticosteroids operate as a switch between memory systems. J Cogn Neurosci 22: $1362-1372$.

Sheskin DJ. 2011. Handbook of parametric and nonparametric statistical procedures. 5th ed. Chapman and Hall/CRC, Boca Raton, FL.

Smith KS, Virkud A, Deisseroth K, Graybiel AM. 2012. Reversible online control of habitual behavior by optogenetic perturbation of medial prefrontal cortex. Proc Natl Acad Sci 109: 18932-18937.

Squire LR. 2004. Memory systems of the brain: A brief history and current perspective. Neurobiol Learn Mem 82: 171-177.

Sullivan BT, Johnson L, Rothkopf CA, Ballard D, Hayhoe M. 2012. The role of uncertainty and reward on eye movements in a virtual driving task. $J$ Vis 12: 19.

Tolman EC. 1948. Cognitive maps in rats and men. Psychol Rev 55: 189-208.

Tolman EC, Ritchie BF, Kalish D. 1946. Studies in spatial learning; place learning versus response learning. J Exp Psychol 36: 221-229.

Ullman MT. 2004. Contributions of memory circuits to language: The declarative/procedural model. Cognition 92: 231-270.

van der Meer M, Kurth-Nelson Z, Redish AD. 2012. Information processing in decision-making systems. Neuroscientist 18: 342-359.

Wit $\mathrm{S}$ de, Watson $\mathrm{P}$, Harsay HA, Cohen MX, van der Vijver I, Ridderinkhof KR. 2012. Corticostriatal connectivity underlies individual differences in the balance between habitual and goal-directed action control. J Neurosci 32: 12066-12075.

Yin HH, Knowlton BJ. 2004. Contributions of striatal subregions to place and response learning. Learn Mem 11: 459-463.

Youmans RJ, Ohlsson S. 2008. How practice produces suboptimal heuristics that render backup instruments ineffective. Ergonomics 51: 441-475.

Zola-Morgan S, Squire LR. 1985. Medial temporal lesions in monkeys impair memory on a variety of tasks sensitive to human amnesia. Behav Neurosci 99: 22-34.

Received April 1, 2013; accepted in revised form July 30, 2013. 


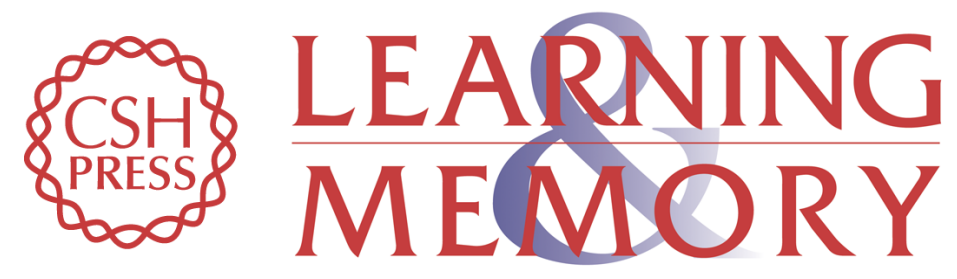

\section{A secondary working memory challenge preserves primary place strategies despite overtraining}

Robert S. Gardner, Michael R. Uttaro, Samantha E. Fleming, et al.

Learn. Mem. 2013, 20:

Access the most recent version at doi:10.1101/Im.031336.113

\section{Supplemental http://learnmem.cshlp.org/content/suppl/2013/09/25/20.11.648.DC1 Material}

References This article cites 59 articles, 11 of which can be accessed free at: http://learnmem.cshlp.org/content/20/11/648.full.html\#ref-list-1

Creative This article is distributed exclusively by Cold Spring Harbor Laboratory Press for the Commons first 12 months after the full-issue publication date (see

License http://learnmem.cshlp.org/site/misc/terms.xhtml). After 12 months, it is available under a Creative Commons License (Attribution-NonCommercial 3.0 Unported), as described at http://creativecommons.org/licenses/by-nc/3.0/.

Email Alerting Receive free email alerts when new articles cite this article - sign up in the box at the Service top right corner of the article or click here. 